\title{
Clinical Data Validated Mathematical Model for Intermittent Abiraterone Response in Castration-Resistant Prostate Cancer Patients
}

\author{
Justin Bennett ${ }^{1}$, Xixu Hu${ }^{2}$, Karissa Gund ${ }^{3}$, Jingteng $\mathrm{Liu}^{1}$, and \\ Anya Porter ${ }^{4}$
}

${ }^{1}$ School of Mathematical and Statistical Sciences, Arizona State University, 901 S. Palm Walk, Tempe, AZ 85287-1804, USA

${ }^{2}$ Department of Statistics and Finance, University of Science and Technology of China, 96 Jinzhai Road ,Hefei, Anhui, 230026, China

${ }^{3}$ Ira A. Fulton Schools of Engineering, Arizona State University, 699 S. Mill Ave, Tempe, AZ 85287-9309, USA

${ }^{4}$ Computer Science Department, Harvey Mudd College, 1250 North

Dartmouth Ave, Claremont, CA 91711-5901, USA

\begin{abstract}
Over time, tumor treatment resistance inadvertently develops when androgen deprivation therapy (ADT) is applied to metastasized prostate cancer (PCa). To combat tumor resistance, while reducing the harsh side effects of hormone therapy, the clinician may opt to cyclically alternates the patient's treatment on and off. This method, known as intermittent ADT, is an alternative to continuous ADT that improves the patient's quality of life while testosterone levels recover between cycles. In this paper, we explore the response of intermittent ADT to metastasized prostate cancer by employing a previously clinical data validated mathematical model to new clinical data from patients undergoing Abiraterone therapy. This cell quota model, a system of ordinary differential equations constructed using D roop's nutrient limiting theory, assumes the tumor comprises of castration-sensitive (CS) and castration-resistant (CR) cancer sub-populations. The two sub-populations rely on varying levels of intracellular androgen for growth, death and transformation. Due to the complexity of the model, we carry out sensitivity analyses to study the effect of $\mathrm{c}$ ertain $\mathrm{p}$ arameters o $\mathrm{n}$ their outputs, and to increase the identifiability of each patient's unique parameter s et. The model's forecasting results show consistent accuracy for patients with sufficient data, which means the model could give useful information in practice, especially to decide whether an additional round of treatment would be effective.
\end{abstract}




\section{Introduction}

\subsection{Background}

Prostate cancer is one of the greatest global health concerns and is the second leading cause of men's deaths by cancer in the United States [22]. The growth of advanced prostate tumors is notably linked with its high dependence on androgen, a male hormone developed in the testis and adrenal gland. Unfortunately, androgen levels are not always directly measurable, nor is androgen data always available. However, prostate cells convert androgen to a potent form called dihydrotestosterone (DHT) and bind to the androgen receptors, thus elevating prostate-specific antigen (PSA) levels and leading to growth and survival of the cancer cells. Then the PSA proteins that are produced by the tissue in the prostate could be used as biomarkers for cancerous activity. [10].

Huggins and Hodges Nobel-prize winning studies on castration and metastasized PCa shows androgen deprivation rapidly regresses tumor size [12]. However, many patients with a metastatic form of cancer eventually suffer a relapse. Treatment that effectively kills most of the tumor's cancerous cells may quickly become ineffective when the cells that remain are those resistant to treatment - or can quickly mutate to sustain growth in an androgendeprived environment. In 2006, Bruchovsky et al. used the CS Shionogi model to conclude that androgen deprivation causes a change in cellular phenotype, from CS to CR [5]. It makes sense that lessening selective pressure would be effective in delaying treatment resistance. Taking the patient off treatment for a period of time would potentially allow the overall tumor population to remain sensitive to ADT.

Patients who respond well to Abiraterone show drastically reduced levels of PSA which demonstrates the efficacy of the treatment. This is shown in [9], where clinical trials show that Abiraterone rapidly decreases serum testosterone to castrate levels in non-castrate patients diagnosed with PCa.

In order to combat treatment resistance, intermittent ADT is introduced. Taking the patient off treatment for a period of time potentially allows the overall tumor population to stay sensitive to ADT. Another treatment method, continuous ADT, causes many side effects that diminish the quality of life of the patient. With intermittent ADT, treatment is administered until PSA levels reach a desired threshold before they are taken off treatment. This allows serum testosterone levels to return to normal, which would not occur under continuous ADT. With intermittent ADT, treatment begins again once PSA levels rise to a predetermined level. [8]

\subsection{Modeling}

The development of previous models for hormonal treatment of prostate cancer has paved the way for further research. Jackson [14] introduced a model that described prostate cancer growth using continuous ADT, which inspired further research by Ideta et al. [13] that separates cancer cells into two sub-populations, CR and CS. Portz et al. [20] extends the work of previous models that take intracellular androgen into account, while incorporating 
the use of clinical data to their model. Hirata et al.[11] introduces a piecewise linear model that potentially permits a more accurate fit of the on and off alternations of intermittent ADT. Baez and Kuang [2] formulate a simplification of the Portz et al. model, incorporating the assumption that the level of intracellular androgen is the same as that of body serum androgen. Moreover, the model is further simplified to incorporate a compartment for serum androgen, referred to as model T in Wu et al. [24] and further modified in Phan et al. [19]. For this paper, we focus on Phan et al.'s modification which we will call "Model T," which incorporates androgen production and its diffusion to intracellular androgen. By modeling the factors that come into play with metastasized $\mathrm{PCa}$, such as the cycling involved with intermittent ADT to potentially avoid or reduce resistance, we are able to predict three to four months of treatment progress. Because the treatment is administered in cycles, we explore whether another round of treatment could be effective for the patient.

Although there has been an emergence of mathematical modeling of cancer within the past few decades, the ability to predict and treat cancer is limited. Being able to reliably forecast each patient's next cycle allows clinicians to choose the best treatment options for each patient's given tumor behavior three to four months prior. The difficulty of reliability in forecasting cancer behavior stems from the fact that cancer is the result of a complex interplay between numerous factors, or cellular parameters. Mathematical modeling attempts to analyze the enormous amount of data being produced and extract useful answers by attempting to mimic the behavior of a tumor on a biological level. However, the accuracy of the predictions varies as the forecast increases.

We use the model to fit the data that is described in section 2.2. For this paper, we fit all but the last full cycle of data to the model, and use the rest of the data to test the predictability of the model. In section 2.4, we introduce the weighted error that we employ to prioritizes recent data. The sensitivity analysis, detailed in section 2.5 , is used to reduce uncertainty and increase robustness of individual patient fits. By doing this, we can explore unique parameter dynamics for each patient. This allows us to set up future attempts to draw biological conclusions for parameters assigned to each individual patient. The precision that the model shows for each patient shows promise for optimal treatment options.

Where this paper differs from similar models and model analyses, is the particular use of Aberiterone to treat the patients. Both $\mathrm{Wu}$ et al. and Phan et al. use similar models, but both data sets are from prostate cancer patients treated with Cyproterone acetate or Leuprolide acetate. Moreover, the weighted exponential error is emphasized in our computations - which we compare in section 3.1.2. Phan et al. found relative success with the weighted error metric implemented with their clinical data, so we chose to use the the same metric to patients treated with Abiraterone to explore its practicality and validity. 


\section{Model and method}

\section{$2.1 \quad$ Model T}

Because the authors did not contribute to the formulation of the model used, we simply describe the model and its dynamics. Model $\mathrm{T}$ is a series of differential equations constructed to describe the dynamics of the cancer cells, and is a cell quota model that originates from the mathematical formulations credited to Portz, Kuang and Nagy (PKN) [20]. The PKN model incorporates Droop's limiting nutrient theory [6], which describes a positive saturating relationship between metabolic performance and availability of an element. Specifically, the PKN model regards intracellular androgen as the threshold for cancer sub-population growth. However, model $\mathrm{T}$ differs from the PKN model by the incorporation of a compartment for serum androgen, as well as the addition of an irreversibly treatment-resistant subpopulation [2][19]. This cell quota model contains five variables ( $x_{1}$ for CS cells, $x_{2}$ for CR cells, Q for intracellular androgen, A for serum androgen, P for PSA ), each describing a dynamic that represents a critical role in how androgen, PSA, and the cancerous cell sub-populations interact. The model takes the form:

$$
\begin{aligned}
& \frac{d x_{1}}{d t}=\underbrace{\mu_{1}\left(1-\frac{q_{1}}{Q}\right) x_{1}}_{\text {growth }}-\underbrace{\left(D_{1}(Q)+\delta_{1} x_{1}\right) x_{1}}_{\text {death }}-\underbrace{\lambda(Q) x_{1}}_{\text {transformation }} \\
& \frac{d x_{2}}{d t}=\underbrace{\mu_{2}\left(1-\frac{q_{2}}{Q}\right) x_{2}}_{\text {growth }}-\underbrace{\left(D_{2}(Q)+\delta_{2} x_{2}\right) x_{2}}_{\text {death }}+\underbrace{\lambda(Q) x_{1}}_{\text {transformation }} \\
& \frac{d Q}{d t}=\underbrace{m(A-Q)}_{\text {androgen diffusion } \mathrm{A} \rightarrow \mathrm{Q}}-\underbrace{\frac{\mu_{1}\left(Q-q_{1}\right) x_{1}+\mu_{2}\left(Q-q_{2}\right) x_{2}}{x_{1}+x_{2}}}_{\text {androgen uptake }} \\
& \frac{d A}{d t}=\underbrace{\gamma_{2}+\gamma_{1}\left(A_{0}-A\right)}_{\text {production }}-\underbrace{A_{0} \gamma_{1} u(t)}_{\text {suppression of production }} \\
& \frac{d P}{d t}=\underbrace{b Q}_{\text {baseline PSA production }}+\underbrace{\sigma\left(Q x_{1}+Q x_{2}\right)}_{\text {tumor PSA production }}-\underbrace{\epsilon P}_{\text {degradation }} \\
& u(t)= \begin{cases}1 & \text { on treatment } \\
0 & \text { off treatment }\end{cases}
\end{aligned}
$$


The growth of both the CS cells (1) and CR cells (2) is dependent upon the internal androgen concentration of the sub-population. At any time the intracellular androgen falls below the "cell quota," the subpopulation will decline, causing cellular apoptosis. Transformation to resistant cancerous cells are assumed to be irreversible, hence the transformation will only facilitate CS cells to CR cells. This means that, unlike the PKN model, $x_{2}$ can't transform into $x_{1}$.

Transformation is not a mutation, but rather refers to phenotypical adaptation due to selective pressure of the treatment - hence it is androgen dependent. The transformation rates between different cells are assumed to be the same, and is described in the form of a hill function which describes switching rates between cell phenotypes:

$$
\lambda(Q)=\frac{c K}{Q+K}
$$

Death is characterized by density dependent competition, a result of assuming interspecies effect is negligible in population growth because cells of the same phenotype tend to cluster. The death term is as follows, and describes how sensitive apoptosis is to variation of the cell quota threshold:

$$
D_{j}(Q)=\frac{d_{j} R_{j}}{Q+R_{j}} j=1,2
$$

A comprehensive derivation of death and transformation in this model can be found via Phan et al., Baez et al. and Morken et al. [2][16][18].

Androgen uptake is based on ecological stoichiometry and Droop's law. The formulation comes from the conservation of androgen as it moves in and out of the tumor. Details regarding uptake in this model can be found in Baez et al. [2].

Intracellular androgen is described by the diffusion of serum androgen into the cell. The unit step function, $u(t)$, characterizes the on and off cycles of treatment when a patient is on intermittent androgen deprivation therapy, and describes the suppression of serum androgen production. Mathematically, a value of 1 (when the patient is on treatment) will effectively reduce production of $A$. A value of 0 (when the patient is off treatment) will eliminate deprivation of $A$ production.

As listed on Table 1, Model T has 19 biological parameters and 5 initial conditions with biological ranges obtained from previous literature [20][24]. The units for this model, along with specific definitions of each variables for the differential equations are established in Phan et al. [19]. Phan et al.'s assumption that serum androgen and intracellular androgen are approximately the same prior to treatment would have been used when selecting initial $\mathrm{Q}(0)$ and $\mathrm{A}(0)$ for this model [19]. This assumption was made with available androgen data. However, the data provided to us by Phoenix's Mayo Clinic did not include androgen levels, but PSA levels instead. The previous assumption inspired a similar approach by setting $Q(0)$ to 40-50 percent of initial PSA, and $A(0)$ to roughly 60-70 percent of initial PSA. We recommend further exploration when modeling without androgen data. 


\begin{tabular}{|c|c|c|c|}
\hline Param & Description & Range & Ref. \\
\hline$\mu_{1}$ & max proliferation rate (CS cells) & {$[0.001,0.09]\left[\mathrm{day}^{-1}\right]$} & {$[3]$} \\
\hline$\mu_{2}$ & max proliferation rate ( $\mathrm{CR}$ cells) & {$[0.001,0.09]\left[\right.$ day $\left.^{-1}\right]$} & {$[19]$} \\
\hline$q_{1}$ & min CS cell quota & {$[0.41,1.73][\mathrm{nmol} / \mathrm{L}]$} & {$[17]$} \\
\hline$q_{2}$ & min $\mathrm{CR}$ cell quota & {$[0.01,0.41][\mathrm{nmol} / \mathrm{L}]$} & estimated \\
\hline $\mathrm{b}$ & baseline PSA production rate & {$[0.0001,0.1][\mu \mathrm{g}][\mathrm{nmol}]^{-1}[\text { day }]^{1}$} & {$[20][2]$} \\
\hline$\sigma$ & tumor PSA production rate & $\left.[0.001,1][\mu \mathrm{g}][\mathrm{nmol}]^{-1}[\mathrm{~L}]^{-1}\right][\text { day }]^{-1}$ & ad hoc \\
\hline$\epsilon$ & PSA clearance rate & {$[0.0001,0.1][\text { day }]^{-1}$} & {$[20][2]$} \\
\hline$d_{1}$ & max CS cell death rate & {$[0.001,0.09][\text { day }]^{-1}$} & {$[3]$} \\
\hline$d_{2}$ & max $C R$ cell death rate & {$[0.01,0.09][\mathrm{day}]^{-1}$} & {$[19]$} \\
\hline$\delta_{1}$ & density death rate (CS cells) & {$[1,90][\mathrm{L}]^{-1}[\text { day }]^{-1}$} & {$[2]$} \\
\hline$\delta_{2}$ & density death rate ( $\mathrm{CR}$ cells) & {$[1,90][\mathrm{L}]^{-1}[\text { day }]^{-1}$} & {$[19]$} \\
\hline$R_{1}$ & CS death rate half-saturation & {$[0,3][\mathrm{nmol} / \mathrm{L}]$} & {$[2]$} \\
\hline$R_{2}$ & $\mathrm{CR}$ death rate half-saturation & {$[1,6][\mathrm{nmol} / \mathrm{L}]$} & {$[7]$} \\
\hline $\mathrm{c}$ & maximum mutation rate & {$\left[10^{-5}, 10^{-4}\right][\text { day }]^{-1}$} & {$[20][7]$} \\
\hline $\mathrm{K}$ & mutation rate half-saturation level & {$[0.8,1.7][\mathrm{nmol}][\mathrm{L}]^{-1}$} & {$[20]$} \\
\hline$\gamma_{1}$ & primary androgen production rate & {$[0.008,0.8][\mathrm{day}]^{-1}$} & [13] \\
\hline$\gamma_{2}$ & secondary androgen production rate & {$[0.001,0.1][\text { day }]^{-1}$} & estimated \\
\hline $\mathrm{m}$ & diffusion rate from $\mathrm{A}$ to $\mathrm{Q}$ & {$[0.01,0.9]$} & [19] \\
\hline$A_{0}$ & maximum serum androgen level & {$[27,35][\mathrm{nmol}][\mathrm{L}]^{-1}$} & {$[2][13][5]$} \\
\hline$x_{1}(0)$ & initial population of CS cells & {$[0.009,0.02]$} & {$[5]$} \\
\hline$x_{2}(0)$ & initial population of CR cells & {$\left[10^{-5}, 10^{-4}\right]$} & {$[5]$} \\
\hline $\mathrm{Q}(0)$ & initial intracellular androgen & $P(0) *[0.4,0.5]^{1}$ & estimated \\
\hline $\mathrm{A}(0)$ & initial serum androgen & $P(0) *[0.6,0.7]^{1}$ & estimated \\
\hline $\mathrm{P}(0)$ & initial PSA level & initial & given \\
\hline
\end{tabular}

Table 1: Estimated biologically realistic ranges of the parameters for Model T. Q $(0)$ and $\mathrm{A}(0)$ were calculated as ratios of the clinical data we were given.

\subsection{Data Description}

The data is from 41 patients at Phoenix, Arizona's Mayo Clinic. Table 2 provides an excerpt of the data, particularly patient 2. Included in this data were the measured PSA amounts, the date the measurement was taken and the patient number. No direct androgen data was provided and the date measurements were converted to time elapsed (in days) between data points. This allowed us to create data points from a beginning treatment date of 0 . No information was provided to ascertain whether the patient was on or off treatment, so the authors were required to manually input this in the data so that the model could kick on or off the suppression of production as described by equations (4) and (6) in section 2.1. The treatment was determined to be on when PSA levels were decreasing, and off when PSA 
levels were increasing. A new cycle was counted when the PSA reached a new relative max before decreasing. Careful consideration of cycle count allowed us to cut the model off with one full cycle remaining. The remaining data was left to be compared to the forecast.

While coding the model in MATLAB, we used the patient data as a reference to create unique fittings for each patient.

Table 2: Patient 2's reference data

\begin{tabular}{|c|c|c|c|c|}
\hline Patient & Time Elapsed & PSA Level & Cycle & On/Off \\
2 & 0 & 13.8 & 1 & 1 \\
\hline 2 & 90 & 0.19 & 1 & 0 \\
\hline 2 & 226 & 0.61 & 1 & 0 \\
\hline 2 & 358 & 6.98 & 1 & 0 \\
\hline 2 & 482 & 2.4 & 1 & 0 \\
\hline 2 & 611 & 13.8 & 1 & 0 \\
\hline 2 & 744 & 4.9 & 1 & 0 \\
\hline 2 & 1000 & 5 & 1 & 0 \\
\hline 2 & 1084 & 7.4 & 1 & 0 \\
\hline 2 & 1109 & 7.8 & 2 & 1 \\
\hline 2 & 1150 & 6.6 & 2 & 1 \\
\hline 2 & 1178 & 5.4 & 2 & 1 \\
\hline 2 & 1246 & 0.1 & 2 & 1 \\
\hline 2 & 1331 & 0.23 & 2 & 0 \\
\hline 2 & 1416 & 0.1 & 2 & 0 \\
\hline 2 & 1529 & 0.13 & 2 & 0 \\
\hline
\end{tabular}

\subsection{Parameter Estimation}

To fit the patient's data, MATLAB's ODE45 function was used to solve our system of differential equations. To obtain parameter estimates, we optimized the parameters using MATLAB's fmincon function. This tool implements an interior point algorithm that minimizes the objective function within the boundaries defined by parameters from Table 1. Because local optimization algorithms are highly dependent on initial guess, we use the same initial conditions across all patients for simplicity. This is mostly due to the time constraints of the REU.

Prior to Phan et al.'s adaptation of this model, the error function was calculated as the sum of squared means, e.g., the MSE method to fit the data. For this model, we attempted to fit the data using the MSE method. However, many unsatisfying data fits of our patients with high PSA error motivated the use of an error correction (section 2.4). Specifically, fmincon's mean-squared-error forecasts for patients would measure much higher than the actual data points due to high PSA peaks when off of treatment. Phan et al. [19] considers the treatment administered over time and the effect time has on the fit. Comparing the 
mean-squared-error with a weighted error showed that the latter provided a superior fit in many situations where clinical data was used.

\subsection{Weighted Error}

Intermittent ADT is characteristically cyclic with sharp peaks. This presents a problem when forecasting data. In particular, a uniform mean error tool would cause the forecast to be underestimated because of the quantity of small PSA data points. Phan et al. implements a weighted error metric that puts an emphasis on recent data while controlling the weight contributions pre and post PSA level peaks [19]. The weighted least square method in exponential form is as follows:

$$
\operatorname{error}_{i=1,2}=\frac{\sum(P S A-D a t a)^{2} e^{-\alpha_{i}\left(t_{f}-t_{0}\right)}}{N_{i}},
$$

where $\alpha$ is the weight as a ratio between zero and one, $N_{i}$ is the number of PSA data points for data set $i, t_{f}$ is the final time of the forecast treatment period, $t_{0}$ is the time of the observed PSA data point, PSA is the PSA level according to the model at time t, and Data is the actual data point at time t. The higher that $\alpha$ is, the lower the weight is that is applied to the earliest data points. The exponential form of the least square error rapidly decreases the weight to combat losing earliest data points by sectioning the data into multiple sets. In Phan et al. [19], the sets were divided pre and post peak levels of PSA. However, because of the lack of cycles for some patients, as well as inconsistent data, we decided to employ the weight division one half cycle prior to the beginning of the forecast - this provided consistent and accurate fits despite the somewhat inconsistent data. Similar to Phan et al, we divided the minimized error function into two parts:

$$
\text { Error }=c_{1} \text { error }_{1}+c_{2} \text { error }_{2}
$$

where $c_{1}$ and $c_{2}$ are the weighted contributions of error from both prior and post PSA peak level, such that $c_{1}+c_{2}=1$ for consistent results. In general, $c_{1}=0.5=c_{2}$ and $a_{1}=0.01=$ $a_{2}$. Because each model is precisely unique to different patients, the value of $c_{1}, c_{2}$ and $\alpha$ will always be different. The objective function that fmincon minimizes is the sum of squared PSA and androgen residuals:

$$
\text { Objective }_{M S E}=\text { Error }_{M S E, P S A}+\text { Error }_{M S E, \text { androgen }}
$$

\subsection{Sensitivity analysis}

In general, sensitivity analysis is implemented to improve the quality of a model. In the interest of identifying precise fits for each patient, sensitivity analysis is used to reduce uncertainty in each of the patient's unique fit. To understand the effect that changing 
a parameter has on the patient's dynamics, we varied the value by a small amount and measured change in the effect at a particular time. For this paper, we use the one factor at a time (OAT) approach [21]:

$$
\frac{\partial x}{\partial p} \cdot \frac{p}{x}
$$

where the partial derivative is the sensitivity coefficient, $p$ is the parameter, and $x$ is the variable being analyzed. We use this method to investigate the behavior of $x$ by a fixed fraction of the parameter's mean value for that patient. By using this method, we identified the most insensitive parameters and converted them to constants, reducing uncertainty in remaining parameters and improving the fit and forecast of individual patients. Since there is a large amount of variability between patient data, it seems reasonable to assume there is no uniform parameter sensitivity. Due to time constraints of the REU in which this analysis was performed, we were unable to investigate biological implications from parameter sensitivity. Instead, we made observations of parameter sensitivity coefficients from a patient with a particularly good forecast (patient 13) and a particularly undesirable forecast (patient 17).

\section{Results}

Our results utilize a qualitative approach by visually comparing parameter sets to interpret the dynamics of individual fits of each patient, and the differences in patient forecasts. The differences we look for are the sensitivity of the parameters used in the model, and patterns in the fitting that could imply model error or give insight to cancerous activity.

\subsection{Fitting and forecasting of androgen and cell population}

Figure 1 shows a comparison of four patients, where we can eyeball the fit of the model to the PSA data. Our intention was to explore whether we can use the model to reliably predict a practical time frame of intermittent ADT progress in advance, i.e. three to four months. Ideally, we would like to predict whether another round of treatment would be meaningful. In other words, would an additional round of hormone treatment effectively reduce PSA levels? There are some spikes or deviations from the data points, likely caused by outliers in the data or the distribution of error weight. Even with the deviations and spikes, the forecast remains consistent with PSA trends, with some forecasts projecting well beyond the three to four months that we were looking to project. 

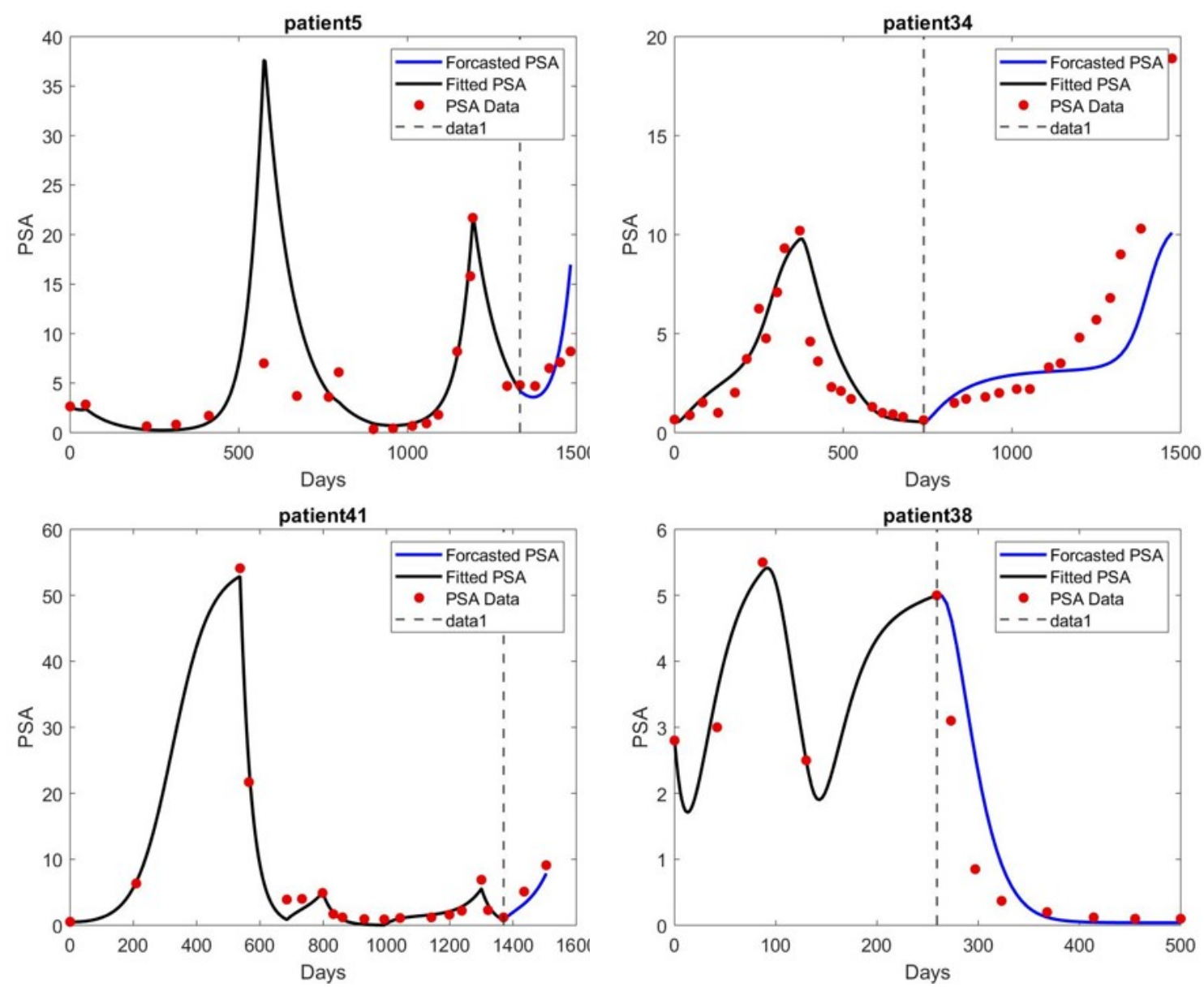

Figure 1: Model T (black line) fits to the patient's PSA levels (the red dots) to train the model to forecast (blue line) the last half cycle. Data1 (dashed line) is where the forecast begins.

On patient 5, we notice the model spikes around day 500 up to roughly 40 PSA. We could possibly infer the model's attempt to compensate for the higher weight closer to the forecast, which shows a spike around day 1200. This is also apparent in the forecast of patient 5 which shows the same trend as the PSA data, but spikes much higher. Patient 38's initial PSA levels vary in a way that could imply that treatment may have not started until around day 100. A logical reason could be the patient switching between alternative medications, however this likely could have been addressed if more data had been available from the beginning up to the forecast. With patient 34, the prediction does not seem to fit very well. It seems that patient 34 was not provided with sufficient training data for the forecast to be accurate. In other words, patient 34's PSA data prior to the forecast should have been comprised of more cycles (ideally 2.5, as explained in section 3.1.1) to cause a better fit forecast. 


\subsubsection{Comparison of full-cycle forecasts}

Some patient datasets had enough data to forecast a full cycle, while other patient datasets were either inconsistent, or did not have enough data to constitute 2.5 cycles prior to forecast. We observed that a full-cycle forecast could not be consistently predicted when less than 2.5 cycles of PSA data were available. When comparing patient figures, the forecast of patient 17 seemingly deviates from the data. To accurately predict a full cycle of intermittent ADT trends in advanced prostate cancer, it seems that model $\mathrm{T}$ needs at least 2.5 cycles before a more accurate prediction can be forecasted. The goal was to forecast reliably for three to four months, which it does not quite do with patient 17 as accurately as a clinician may like. In contrast, patient 13 has 2.5 intermittent ADT cycles of PSA data and shows a promising full-cycled prediction.
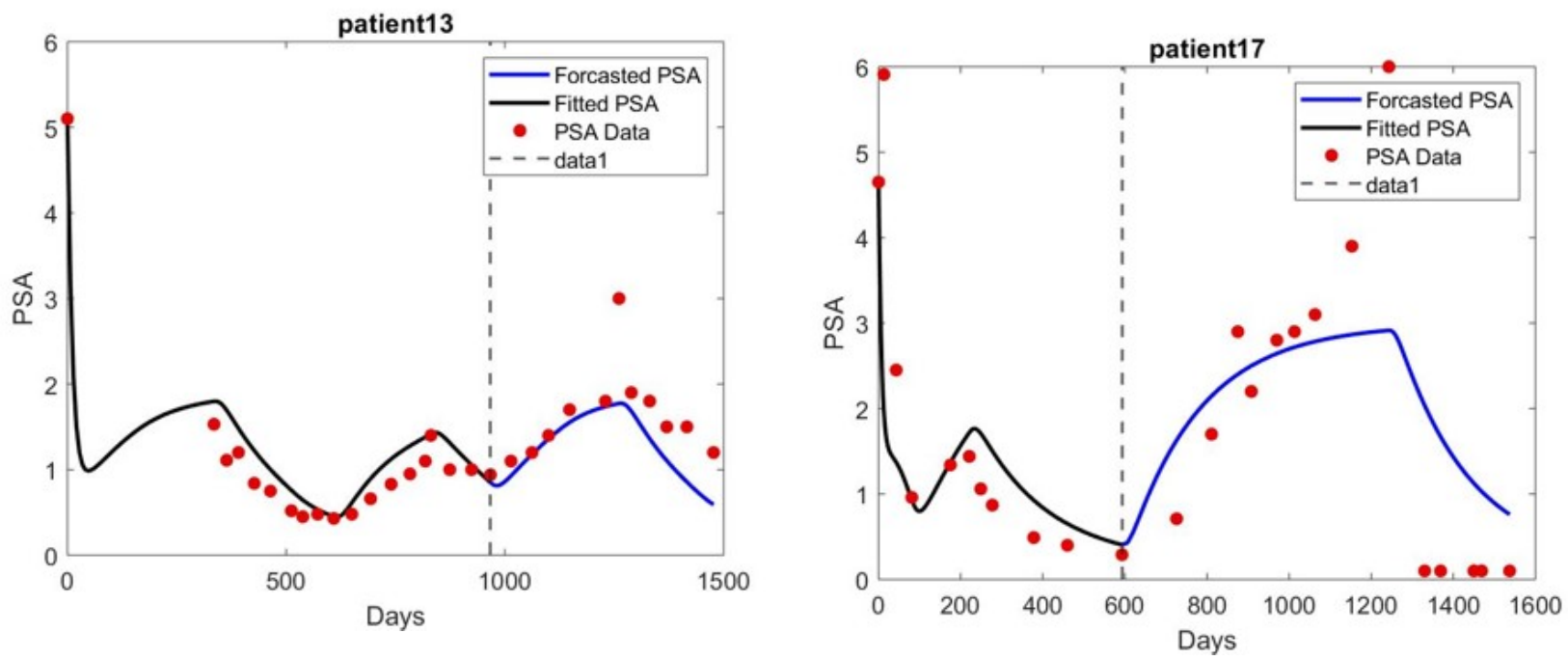

Figure 2: PSA data and model fits and predictions for patients 13 and 17. Model T seems to have sufficient data to reasonably forecast a full cycle for patient 13. The lack of data and lack of intermittent ADT cycles seems to cause the model's forecast to deviate from patient 17 's actual data.

\subsubsection{Comparison of Mean Squared Error and Weighted Exponential Error}

Mean squared error method is commonly used to minimize error in models across many scientific disciplines. The problem with using this method in our case, is that clinical data may not be very consistent, thus causing the data points to be a bit erratic. For example, Figure 3 shows a large gap in data between the first two points. By comparing the mean squared method to the weighted error that we employed, we can argue that a weighted error method may be superior, at least when using clinical data. Phan et al. uses weighted error in a similar clinical application using intermittent ADT [19]. By using weighted error in this application, we can verify prediction accuracy with the use of the drug Abiraterone. 

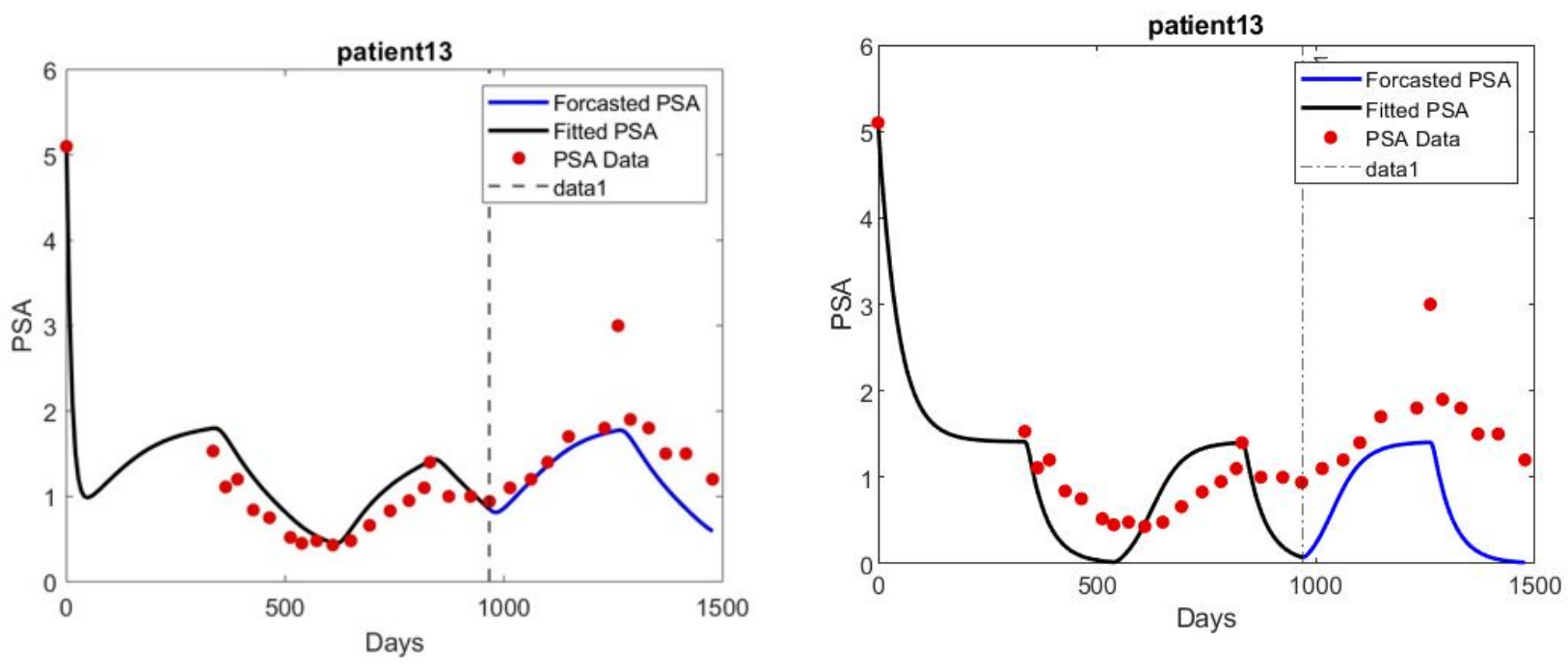

Figure 3: PSA data and model fits and predictions for patients 13. The weighted error method (left) has a significantly more accurate fit than the mean squared error method (right).

A reason the mean squared fit may fluctuate in such a manner may be because it takes the average of all the data points into account when finding an expected value. In contrast, the weighted error puts far less weight on earlier data points. Notice that there are two data points (at roughly day 0 and day 1300) that deviate far from the other data points. Also, notice the relatively large gap between day 0 and the next data point. This large gap likely plays the greatest factor in the large fluctuations in the MSE method use on patient 13's data.

\subsection{Sensitivity Results}

Analyzing the sensitivity of parameters can be beneficial for making connections to the biological causes of certain phenomena. Here, the vertical axis of figures 4 through 8 are the normalized sensitivity coefficients. We observed many differing sensitivity coefficients between the parameters of patients 13 and 17. This is expected, because each of the patient's data will have a unique fit. However, we observed similar sensitivity coefficients between patients 13 and 17 . For example, both patients showcased that $x_{2}$ had a positive sensitivity coefficient with respect to $\mu_{2}$. This is expected because a small positive change in CR cell populations would imply the expectation of CR cells to take over a hormonally failing patient [19].

Parameters $c, K, q_{2}$, and $R_{2}$ were kept constant for both patients. Some noticeable agreed correlations between patient sensitivity coefficients are between $Q$ and $\gamma_{1}, x_{2}$ and $\mu_{2}$, and $x_{2}$ and $\delta_{2}$. Also, $\gamma_{1}$ has a negative coefficient with respect to all of the variables in both figures. 
From the sensitivity results, we show that patient 13's $x_{1}$ has a high positive relationship with $\mu_{1}$, and similarly $x_{2}$ has a high positive relationship with $\mu_{2}$. A reason for this could be the effect that the proliferation rate of the castration-sensitive and castration-resistant subpopulations have on their respective cancer cells' sub-population. This relationship makes biological sense, because the growth of these variables is contingent upon the rate of the cells' reproduction.

The following are comparisons between patient 13 (left) and patient 17 (right). Due to time constraints of the REU, these were the only two sensitivity analyses conducted.

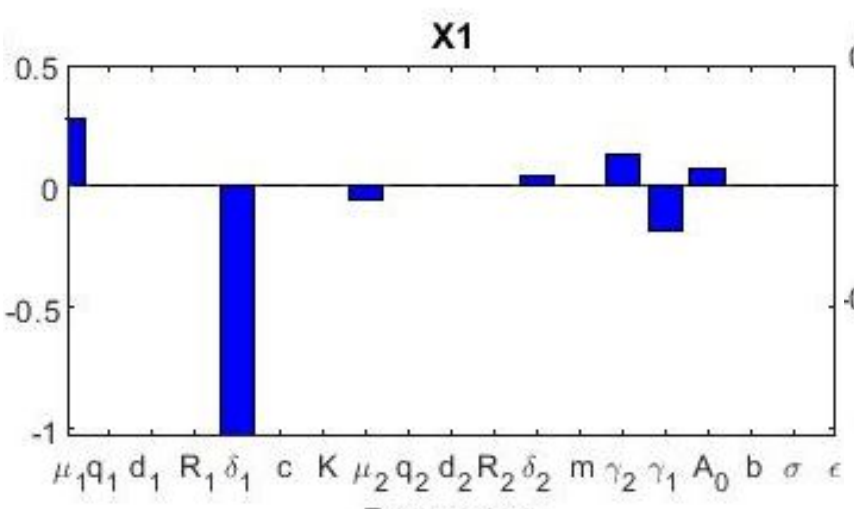

Parameters

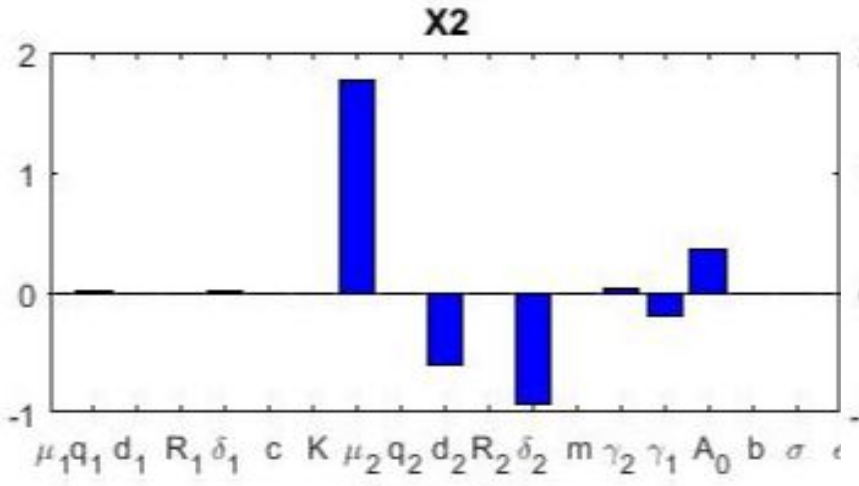

Parameters

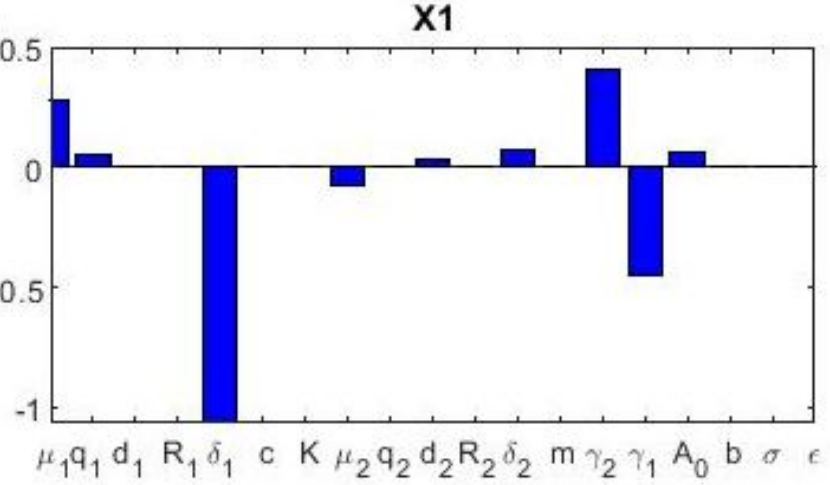

Parameters

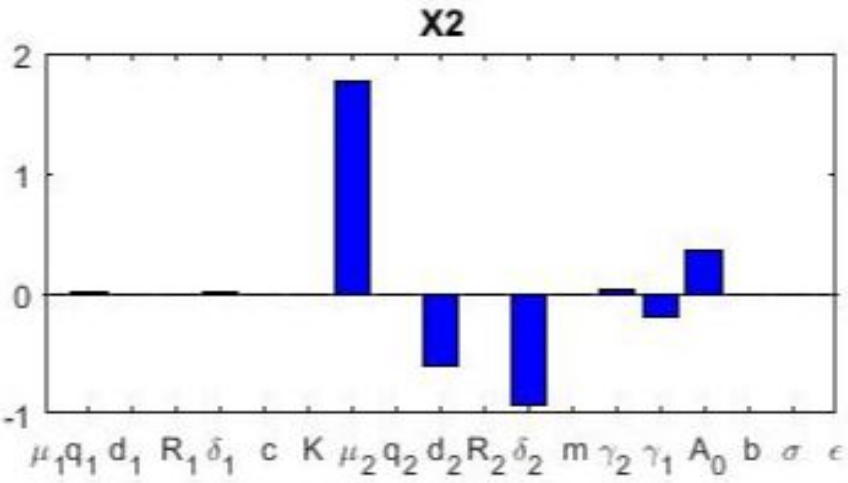

Parameters

Figure 4: The castration-sensitive sub-populations (top) show consistent sensitivity across two different patients. The castration-resistant sub-population (bottom) shows $d_{2}$ to be more sensitive in patient 13, than with patient 17. Patient 17's sensitivity analysis shows $m, \gamma_{1}$, $\gamma_{2}$ and $\alpha_{0}$ have very little sensitivity with respect to the castration-resistant sub-population, $x_{2}$. 


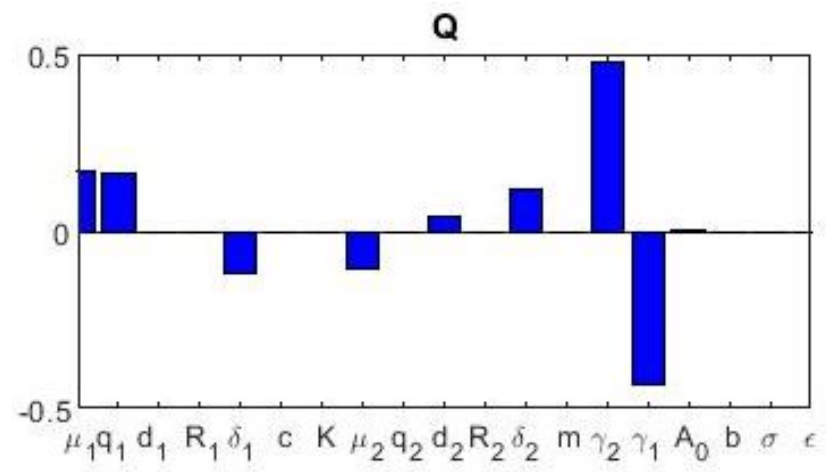

Parameters

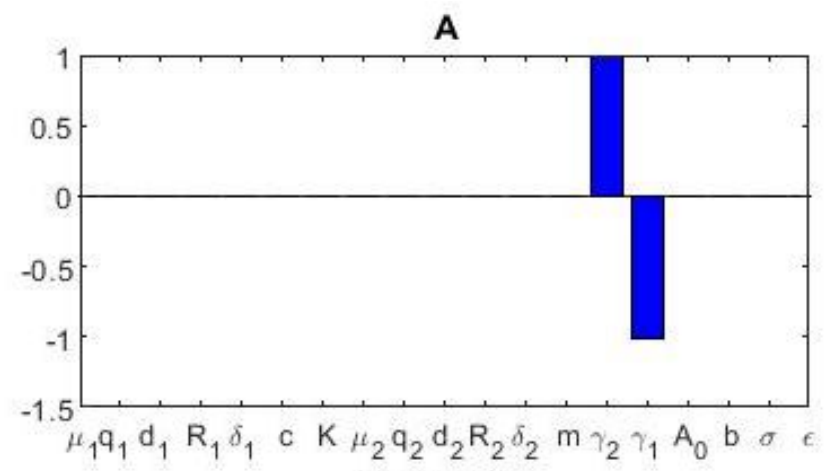

Parameters

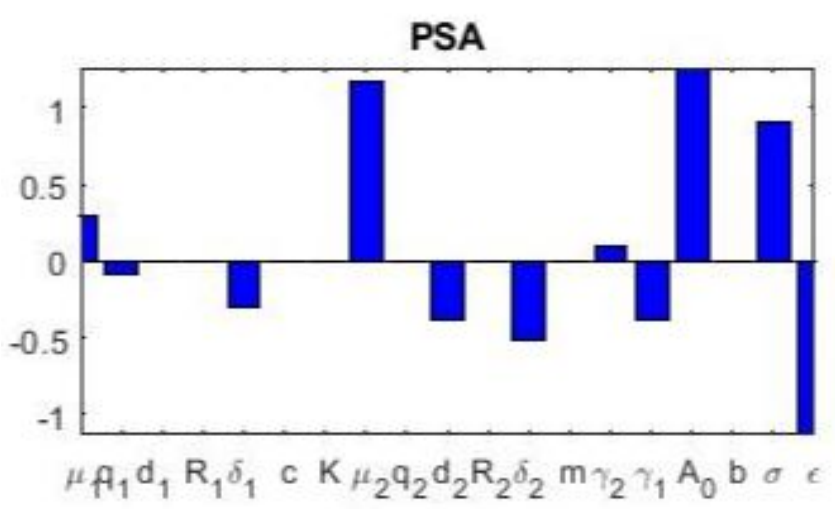

Parameters

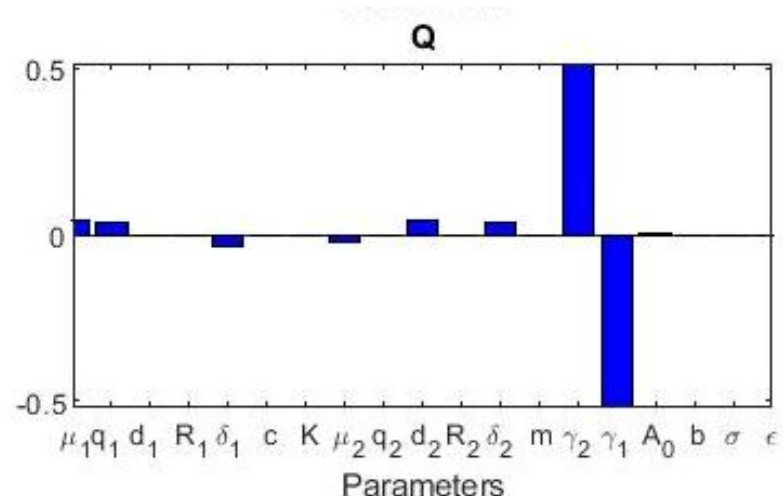

A

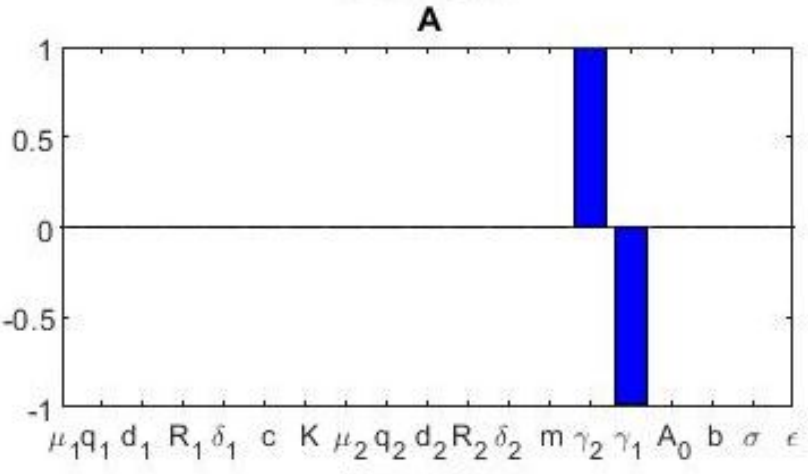

Parameters

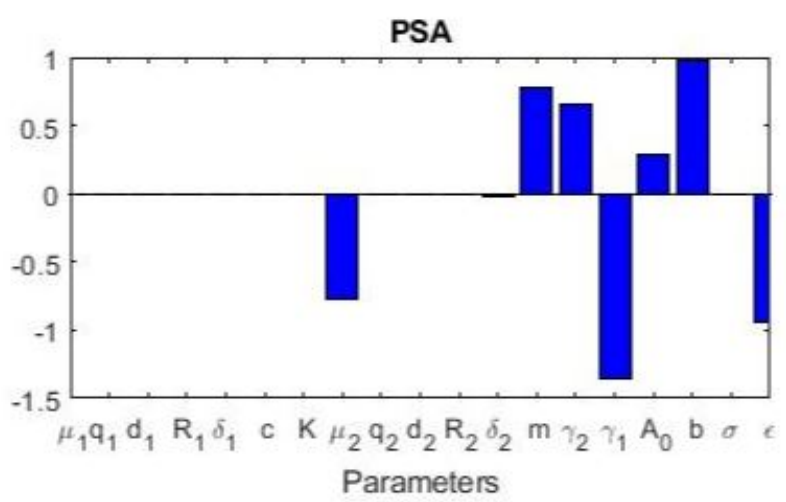

Figure 5: Intracellular androgen (top) displays consistent sensitivity of the parameters across both patients 13 and 17 . Serum androgen (middle) also displays consistent sensitivity of the parameters across both patients 13 and 17. There are differing sensitivity of parameters between patient 13 and patient 17's PSA parameters (bottom). 


\section{Discussion}

The focus of the implemented work was to extend the efforts of Phan et al. by applying the modified version of Baez and Kuang's model, Model T, to a different set of clinical data and a different hormone, Aberiterone. We used the sensitivity analysis to find parameters unique to each patient to minimize error between PSA data and model T. Due to the sporadic nature of data reporting and/or data collecting, we had erratic data to work with. Not every patient's data worked with the model because of the lack of cycles, lack of data points, large gaps between data points, or inconsistent data points. In fact, of the forty-one patients we obtained data for, only ten provided sufficient data to be used with the model. Specifically, the fit of the other patient's datasets never captured the cyclic trend of intermittent ADT in metastasized PCa. However, the clinical data from the ten patients should permit valuable insight for potential clinical usage and future work. As for the concern of validating a reliable three to four month forecast, the fittings and forecasts of model T in Section 3.1 show that it is possible when enough data cycles are available to appropriately train the model to predict the cyclic nature of intermittent ADT. The patient figures of all ten forecasts have been added to appendix A

We used an exponential weighted error which Phan et al. [19] found to be superior to mean square error in the application of intermittent ADT. We found this particular method for reducing error useful for modeling clinical data associated with intermittent ADT and Aberiterone. The weighted error approach controls the weight pre and post peak PSA levels and gives more recent data a higher weight. We show that weighing the later data points contribute to a better fit to the clinical data, and that the earlier data points do not necessarily contribute as much to the forecast, which is useful when updating the model with newly received data. This is not to say that the earlier data is not significant. Figure 3 shows a direct comparison of mean squared versus weighted exponential, and provides valuable insight on the possibility of continuing the weighted error method for the fitting of clinical data in future work involving intermittent ADT.

Intermittent ADT is cyclic, which means the model must incorporate the cyclic nature of intermittent ADT when making predictions. As mentioned before, we observed that at least 2.5 cycles of patient data may be necessary before an accurate full-cycle prediction could be made. However, it is recommended that this be explored in future work. If this is the case, then a certain amount of therapy must have already been applied before this model could make a reasonably accurate full-cycle prediction for each specific patient, preventing full-cycle forecasts from being predicted prior to initial treatment. However, it should be noted that this model has been used previously with completely different hormones [19]. In other words, this application could contribute to future work in generality of intermittent ADT predictions for patients with advanced PCa.

The outputs of varied patient parameters and error weights likely are a result of the uniqueness of each patient's cancer dynamics. Our sensitivity analysis allowed us to reduce the dimension of the parameter space and increase identifiability, which resulted in unique fits for each patient. This is a significant factor in the context of precision medicine, a 
key concept in patient-specific modeling and forecasting. In Figure 4 , we noticed that $\gamma_{1}$ (primary androgen production rate) had a negative association with both $x_{1}$ and $x_{2}$. There is an underlying assumption that androgen is depleted as fast as it is produced. Androgen level goes down at rate $\gamma_{1}$ when the patient is on treatment, and will go up at rate $\gamma_{1}$ while the patient is off treatment. This is convenient to represent the depletion of androgen following treatment. However, in fact, the correlation of $\gamma_{1}$ depends on the time that we select to evaluate the sensitivity coefficient. If we pick a time during the off-treatment interval, the coefficient would be positive. The sensitivity results discussed in Section 3.2 provided some qualitative observations that may be a useful for future work.

\section{Acknowledgement}

This work is supported by the $(\mathrm{AM})^{2}$ program provided by NSF DMS-1757663. We are profoundly grateful to Mayo Clinic for the clinical data set. In addition, the authors would like to thank Dr. Wenbo Tang and Dr. Eric Kostelich for providing valuable discussions and thank our outstanding advisor Dr. Yang Kuang, as well as his research assistant, Tin Phan, for many helpful comments. The code used for this project originated from Dr. Kuang's former PhD student, Javier Baez, and has been edited by Tin Phan, Kyle Nguyen, and Alejandro Martinez before being further edited by the authors of this manuscript. We also thank the referees for their many helpful comments.

\section{References}

[1] P. A. Abrahamsson, Potential benefits of intermittent androgen suppression therapy in the treatment of prostate cancer: A systematic review of the literature, European Urology, 57 (2010), pp. 49-59.

[2] J. BAEZ AND Y. KUANG, Mathematical models of androgen resistance in prostate cancer patients under intermittent androgen suppression therapy, Applied Sciences, 6 (2016), p. 352 .

[3] R. Berges, J. Vukanovic, J. Epstein, M. Carmichel, L. Cisek, D. Johnson, R. Veltri, P. WAlsh, And J. IsAaCs, Implications of cell kinetic changes during the progression of human prostatic cancer, Clinical Cancer Research, 1 (1995), pp. 473-480.

[4] R. Brady-Nicholls, H. Enderling, R. Gatenby, J. Zhang, A. Wang, T. Zhang, T. Gerke, And J. NAGy, Prostate-specific antigen dynamics predict individual responses to intermittent androgen deprivation, Nature communications, (2020).

[5] N. Bruchovsky, L. Klotz, J. Crook, S. Malone, C. Ludgate, W. J. Morris, M. E. Gleave, And S. L. Goldenberg, Final results of the canadian prospective phase ii trial of intermittent androgen suppression for men in biochemical recurrence after radiotherapy for locally advanced prostate cancer, Cancer, 107 (2006), pp. 389-395. 
[6] M. Droop, Some thoughts on nutrient limitation in algae, Journal of Phycology 9, (1973), pp. 264-272.

[7] R. A. Everett, A. M. Packer, And Y. Kuang, Can mathematical models predict the outcomes of prostate cancer patients undergoing intermittent androgen deprivation therapy?, Biophysical Reviews and Letters, 09 (2014), pp. 173-191.

[8] B. J. Feldman and D. Feldman, The development of androgen-independent prostate cancer, Nature Reviews Cancer, 1 (2001), pp. 34-45.

[9] B. A. Gartrell and F. SAad, Abiraterone in the management of castration-resistant prostate cancer prior to chemotherapy, Therapeutic Advances in Urology, 7 (2015), pp. 194-202.

[10] Heinlein, A. Cynthia, Chang, and Chawnshang, Androgen receptor in prostate cancer, Apr 2004.

[11] Y. Hirata, N. Bruchovsky, And K. Ainara, Development of a mathematical model that predicts the outcome of hormone therapy for prostate cancer, Journal of Theoretical Biology, 264 (2010), pp. 517-527.

[12] C. Huggins and C. V. Hodges, Studies on prostatic cancer: The effect of castration, of estrogen and of androgen injection on serum phosphatases in metastatic carcinoma of the prostate, Cancer Research, 1 (1941), pp. 293-297.

[13] A. M. Ideta, G. Tanaka, T. Takeuchi, and K. Ainara, A mathematical model of intermittent androgen suppression for prostate cancer, Journal of Nonlinear Science, 18 (2008), pp. 593-614.

[14] T. JACKSON, A mathematical model of prostate tumor growth and androgen-independent relapse, Discrete and Continuous Dynamical Systems - Series B, 4 (2003), pp. 187-201.

[15] B. L. Maughan and E. S. Antonarakis, Androgen pathway resistance in prostate cancer and therapeutic implications, Expert Opinion on Pharmacotherapy, 16 (2015), pp. 1521-1537.

[16] J. D. Morken, A. Packer, R. A. Everett, J. D. Nagy, and Y. Kuang, Mechanisms of resistance to intermittent androgen deprivation in patients with prostate cancer identified by a novel computational method, Cancer Research, 74 (2014), pp. 36733683.

[17] T. Nishiyama, Serum testosterone levels after medical or surgical androgen deprivation: A comprehensive review of the literature, Urologic Oncology: Seminars and Original Investigations, 32 (2014), pp. 38-e17.

[18] T. Phan, C. He, A. Martinez, and Y. Kuang, Dynamics and implications of models for intermittent androgen suppression therapy, Jan 2019. 
[19] T. Phan, K. Nguyen, P. Sharma, and Y. Kuang, The impact of intermittent androgen suppression therapy in prostate cancer modeling, Applied Sciences, 9 (2018).

[20] T. Portz, Y. KuAng, And J. Nagy, A clinical data validated mathematical model of prostate cancer growth under intermittent androgen suppression therapy, AIP Advances, 2 (2012).

[21] A. Saltelli, K. Chan, and E. M. Scott, Sensitivity analysis, Wiley, 2008.

[22] R. L. Siegel, K. D. Miller, And A. Jemal, Cancer statistics, 2019, CA: A Cancer Journal for Clinicians, 69 (2019), pp. 7-34.

[23] G. Tanaka, Y. Hirata, S. L. Goldenberg, N. Bruchovsky, and K. Aihara, Mathematical modelling of prostate cancer growth and its application to hormone therapy, Philosophical Transactions of the Royal Society A: Mathematical, Physical and Engineering Sciences, 368 (2010), pp. 5029-5044.

[24] Z. Wu, T. Phan, J. Baez, Y. Kuang, and E. Kostelich, Predictability and identifiability assessment of models for prostate cancer under androgen suppression therapy, Mathematical Biosciences and Engineering, 16 (2019), pp. 3512-3536. 
${ }_{402}$ A Patient figures
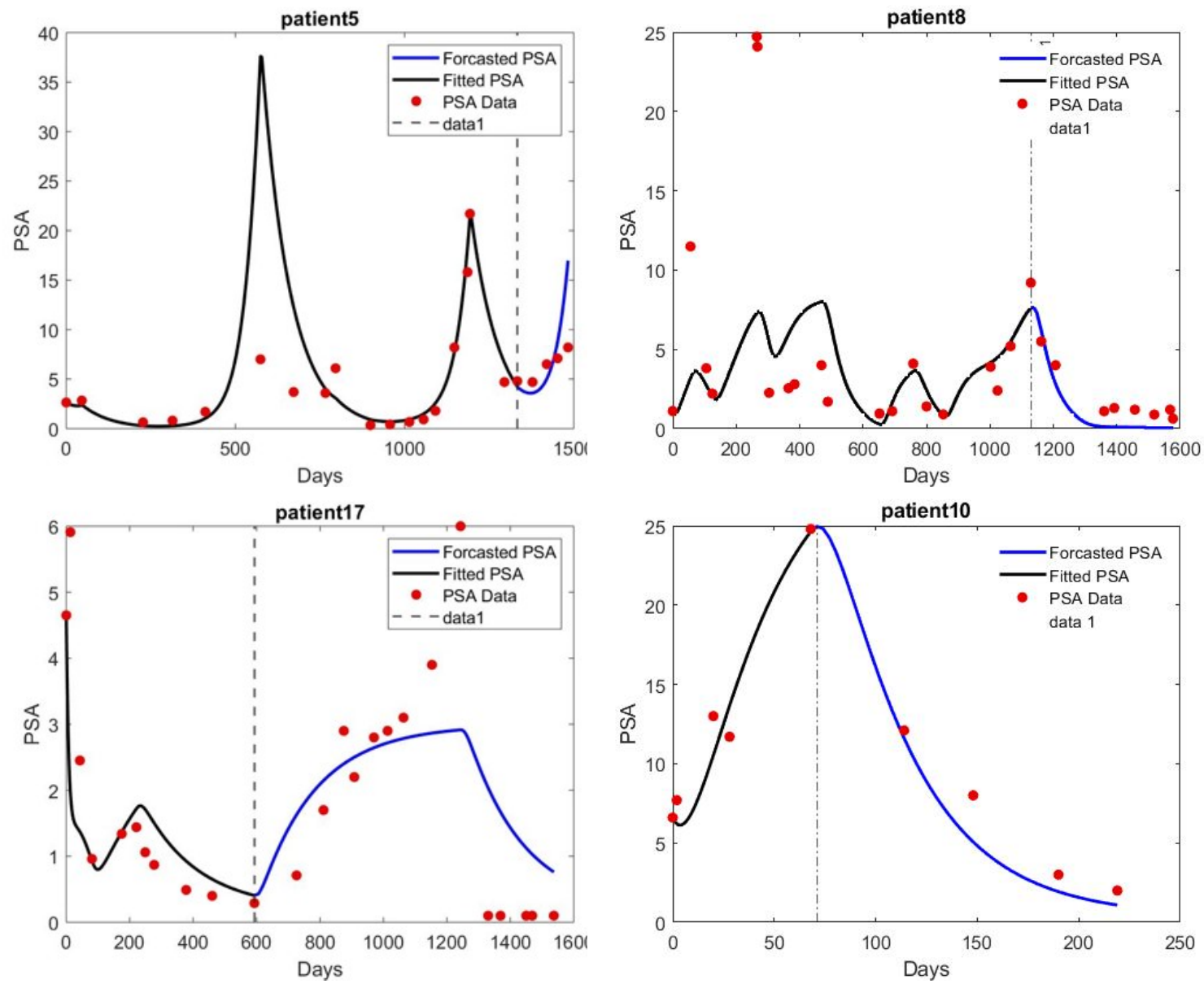

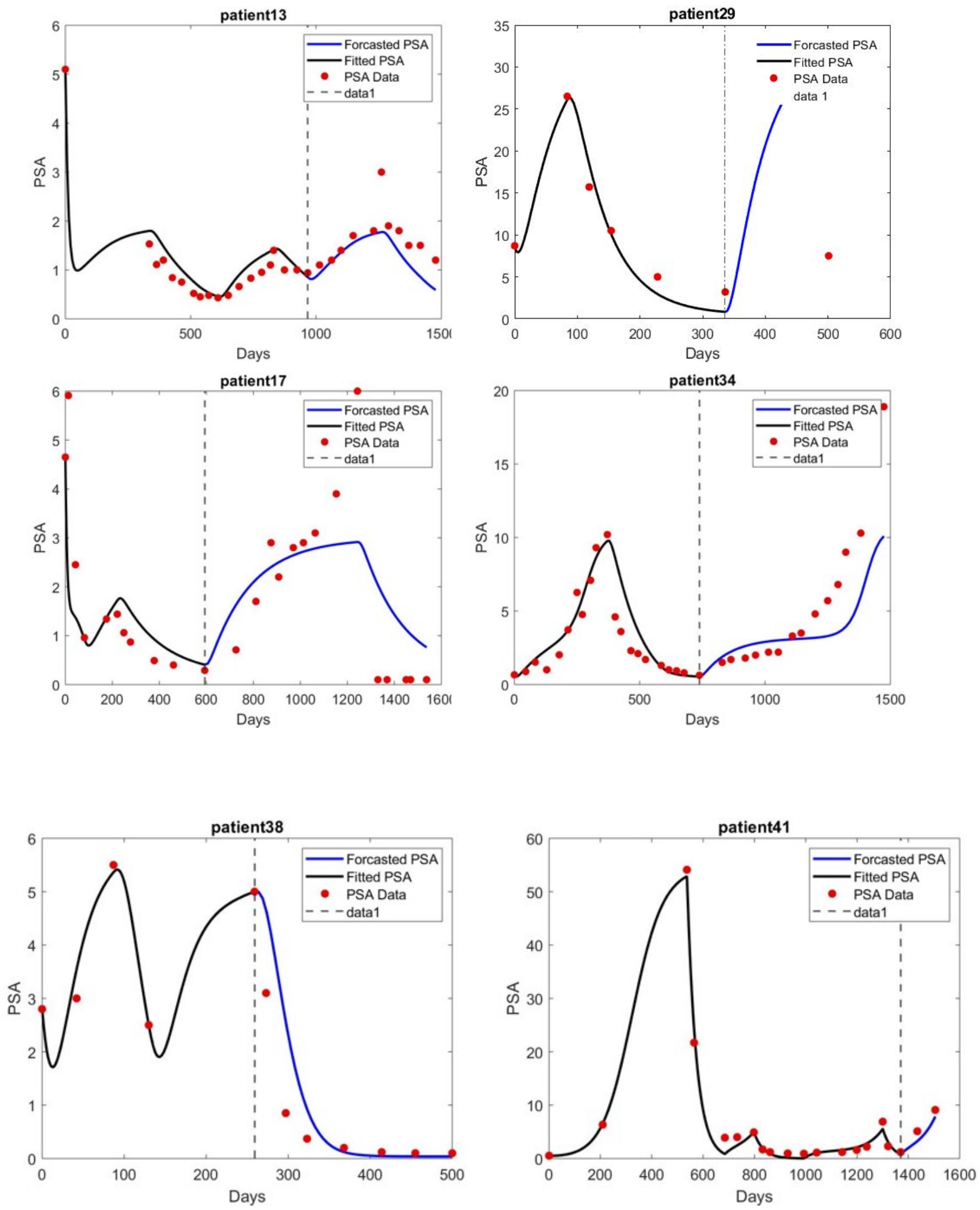